\title{
Oligosaccharide excretion in adult Gaucher disease
}

\author{
J. G. N. De JonG ${ }^{1 *}$, J. M. F. G. Aerts ${ }^{2}$, S. van WeEly ${ }^{2}$, C. E. M. Hollak ${ }^{3}$, \\ J. VAN PELT ${ }^{4}$, L. M. J. VAN WOERKOM ${ }^{1}$, M. L. F. LIEBRAND-VAN SAMBEEK ${ }^{1}$ \\ and R. A. WEVERs ${ }^{1}$ \\ ${ }^{1}$ Laboratory for Neurology and Pediatrics, University Hospital Nijmegen, Nijmegen; \\ ${ }^{2}$ Department of Biochemistry, ${ }^{3}$ Department of Internal Medicine and Hematology, \\ Academic Medical Centre, University of Amsterdam $;{ }^{4}$ Laboratory for Clinical \\ Chemistry, St. Maartens Gasthuis, Venlo, The Netherlands \\ * Correspondence: Laboratory for Neurology and Pediatrics, University Hospital \\ Nijmegen, PO Box 9101, 6500 HB, Nijmegen, The Netherlands
}

MS received 15.4.97 Accepted 18.8.97

\begin{abstract}
Summary: Gaucher disease is a lysosomal storage disease characterized by storage of glucocerebroside due to lysosomal glucocerebrosidase deficiency. Increased urinary excretion of sialyloligosaccharides and mannosylglycoasparagines has been described for two patients with the infantile form of the disease, probably as a consequence of obstruction of lysosomal functioning due to the glycolipid accumulation in lysosomes. By thin-layer chromatography, we found increased urinary oligosaccharide excretion in a series of adult nonneuronopathic patients. Oligosaccharide patterns were comparable between patients and also with the pattern observed in infantile Gaucher disease. Composition was analysed by methanolysis and gas chromatography. Mannose and $\mathrm{N}$-acetylglucosamine are the main carbohydrates in all oligosaccharide bands. A statistically significant correlation was found between oligosaccharide excretion and the severity of the disease expressed as severity score index. Patients treated with enzyme replacement therapy showed a reduction up to $65 \%$ of the original oligosaccharide excretion after 1 year of treatment, comparable with the reduction in spleen volume.
\end{abstract}

Gaucher disease (McKusick 230800) is a lysosomal storage disease due to a deficiency of acid glucocerebrosidase. Storage of the sphingolipid glucocerebroside is primarily in cells of the mononuclear phagocytic system. In the chronic, nonneuronopathic form of the disease (type 1) the main clinical symptoms are hypersplenism with haematological abnormalities (thrombocytopenia, anaemia, leukopenia), hepatomegaly and bone lesions. In the acute (type 2) and subacute 
(type 3) neuronopathic forms the nervous system is also involved (Beutler and Grabowski 1995). Enzyme replacement therapy with modified placental glucocerebrosidase is now applied for the non-neuronopathic form (Barton et al 1991; Beutler et al 1991; Fallet et al 1992; Pastores et al 1993; Hollak et al 1995). Urinary oligosaccharide excretion is increased in lysosomal diseases with deficiencies of enzymes involved in the degradation of oligosaccharide originating from glycoproteins (Thomas and Beaudet 1995). With respect to the sphingolipidoses, oligosaccharide excretion as a direct consequence of the enzyme deficiency is seen in GM1gangliosidosis and Sandhoff disease (Gravel et al 1995; Suzuki et al 1995). Increased excretion of oligosaccharide in Gaucher disease has been described in two patients with the infantile neuronopathic form probably as a consequence of obstruction of lysosomal functioning due to the massive glycolipid accumulation in the lysosomes (Michalski et al 1983). In this paper we describe increased excretion of oligosaccharide in adult non-neuronopathic patients. The amount of excreted oligosaccharides appears to be related to the severity of the disease.

\section{METHODS}

Thin-layer chromatography of oligosaccharides: Urine specimens $(1 \mathrm{ml})$ were desalted and freed from monosaccharides by gelfiltration on $15 \mathrm{ml}$ columns containing Bio-Gel P-2 (Bio-Rad Laboratories, CA, USA). Columns were eluted with deionized water. When fractions of $1 \mathrm{ml}$ were collected, oligosaccharides were detected in fractions 6-12 and monosaccharides, sodium and creatinine in fractions 13-15. The oligosaccharide-containing fractions 6-12 were combined, evaporated to dryness in a Savant AS290 Automatic Speed-Vac concentrator (Savant Instruments, Inc. Farmingdale NY, USA) and dissolved again in the original volume of $1 \mathrm{ml}$. Oligosaccharides were analysed by thin-layer chromatography on silicagel 60 plates (Merck, Darmstadt, Germany, no. 5553) (Blom et al 1983). The amount applied (in $\mu \mathrm{l} / \mathrm{cm}$ ) depended on the creatinine content of the original urine and was calculated by the formula 200/creatinine content (in $\mathrm{mmol} / \mathrm{L}$ ). Thin-layer plates were developed in n-butanol-acetic acid-water $(2: 1: 1$, by vol). A characteristic pattern of eight oligosaccharide bands below the sialyllactose oligosaccharide referent was seen in urines from Gaucher patients. Oligosaccharides, sialyloligosaccharides and glycopeptides were visualized by orcinol, resorcinol and ninhydrin staining, respectively. For the orcinol staining, plates were sprayed with a solution of $0.2 \%$ orcinol (BDH Laboratory Supplies, Poole, UK, no. 29418) in 10\% sulphuric acid and heated for 20 min at $90^{\circ} \mathrm{C}$. The ninhydrin staining was performed as described (Schindler et al 1990) using ninhydrin from Merck (no. 6762). Resorcinol staining was with a $0.2 \%$ resorcinol (Merck no. 7593) solution in 30\% hydrochloric acid. After spraying, the TLC plate was heated between glass plates for $30-45 \mathrm{~min}$ at $100^{\circ} \mathrm{C}$.

Oligosaccharide quantification: A quantitative measure of the oligosaccharide content was obtained by measurement of mannose content of the oligosaccharide fraction (obtained after the Bio-Gel P-2 chromatography) quantified by gas chromatography after methanolysis as described by Chaplin (1986) with some modifications. Volumes 
of 50 and $100 \mu \mathrm{l}$ desalted urines to which $25 \mathrm{nmol}$ of an internal standard (myoinositol) was added were dried by evaporation of the water and by placement in a vacuum desiccator over $\mathrm{P}_{2} \mathrm{O}_{5}$. Methanolysis was performed by incubation with $200 \mu \mathrm{l}$ water-free methanolic $\mathrm{HCl}(0.5 \mathrm{~mol} / \mathrm{L})$ (Supelco, Bellefonte, PA, USA, no. 3-3095) during $24 \mathrm{~h}$ at $80^{\circ} \mathrm{C}$. After neutralization to $\mathrm{pH} 5-6$ (pH test strip) with $\mathrm{Ag}_{2} \mathrm{CO}_{3}$, hexosamines were reacetylated by the addition of $50 \mu \mathrm{l}$ acetic anhydride and an overnight incubation at room temperature. Supernatants obtained after centrifugation were dried by evaporation. Derivatization was by incubation with $60 \mu \mathrm{l}$ $\mathrm{N}, \mathrm{O}$-bis(trimethylsilyl)trifluoroacetamide (BSTFA) (Pierce Europe B.V., Oud Beijerland, The Netherlands, no. 38832) in pyridine $(1: 1, \mathrm{v} / \mathrm{v})$ solution at $60^{\circ} \mathrm{C}$ during $2 \mathrm{~h}$. The trimethylsilylated methylglycosides obtained were quantified by gas chromatography with myoinositol as an internal standard on a HP 5880 gas chromatograph using an Ultra $1 \mathrm{HP}$ column with a length of $25 \mathrm{~m}$, a diameter of $0.2 \mathrm{~mm}$ and a film thickness of $0.11 \mu \mathrm{m}$, both from Hewlett Packard Nederland BV, Amstelveen, The Netherlands. The temperature program was started at $120^{\circ} \mathrm{C}(5 \mathrm{~min})$ followed by a temperature gradient from $120^{\circ} \mathrm{C}$ to $220^{\circ} \mathrm{C}$. The column was regenerated at $295^{\circ} \mathrm{C}$ (5 min). Split ratio was $1: 20$, injector temperature was $250^{\circ} \mathrm{C}$, and the detector temperature was $300^{\circ} \mathrm{C}$. Injection volume was $2 \mu$.

Analysis of oligosaccharide bands: To analyse the composition of the eight oligosaccharide bands, seen on TLC below the sialyllactose reference compound, the corresponding oligosaccharides were isolated from $2.50 \mathrm{ml}$ urine (creatinine value $6.55 \mathrm{mmol} / \mathrm{L}$ ) from a Gaucher patient. The urine was desalted with a $125 \mathrm{mg}$ Duolite mixed bed resin ion exchanger (BDH Ltd, no, MB 5113) essentially as described by de Jong and colleagues (1994). The desalted urine was concentrated to $500 \mu \mathrm{l}$ and applied to two $20 \times 20 \mathrm{~cm}$ TLC plates on two strokes of $15 \mathrm{~cm}$ each. The thin-layer plate was partially stained by spraying with orcinol-sulphuric acid solution $(0.5 \mathrm{~cm}$ at each side of the lanes). Unstained areas corresponding to the eight oligosaccharide bands and the origin were scraped off and oligosaccharides were eluted with water. Part of the nine eluted fractions $(36 \%)$ was used for rechromatography on TLC. Fractions gave one band by orcinol staining. They were also tested with resorcinol (sialyloligosaccharides, sialylglycopeptides) and ninhydrin (glycopeptides) staining. The other part (64\%) was used for analysis of the sugar composition and was therefore methanolysed with methanolic $\mathrm{HCl}$, reacetylated, derivatized with BSTFA, and analysed by gas chromatography as described.

Patients and controls: Twenty-six adult type 1 Gaucher disease patients and one type 2 patient were studied. For all Gaucher type 1 patients a severity score index was established according to the criteria described by Zimran and colleagues (1992). Increases in spleen and liver volumes were calculated by subtracting normal values for liver and spleen $(2.5 \%$ and $0.2 \%$ of body weight, respectively) from measured organ values. Liver and spleen volumes were measured by spiral computed axial tomography as described (Hollak et al 1995).

Enzyme treatment: Adult type 1 Gaucher patients were treated with modified glucocerebrosidase (Ceredase, alglucerase, Genzyme, MA, USA; 1.15 U/kg 3 times a 
week) as described by Hollak and colleagues (1995). Oligosaccharide excretion, liver volume and spleen volume were measured before therapy and after a period of 6 and 12 months.

Chitotriosidase: Chitotriosidase activity in plasma was measured as described by Hollak and colleagues (1994).

Statistical analysis: (Pearson) Correlation coefficients were calculated and tested for their significance by standard procedures. For the calculations, the logarithm of the measured mannose excretion was always used (Keene 1995). All calculations were performed by means of Statistical Analysis System (SAS) procedures.

\section{RESULTS}

Thin-layer chromatography: In TLC patterns from non-neuronopathic patients at least eight orcinol-positive bands were visible in the area below the sialyllactose referent in positions where in the normal control patterns no bands or only weak bands were detectable (Figure 1a and 1b). From these eight bands at least four were recognized as resorcinol (sialic acid)-positive (bands 3,5,6 and 8), both in TLC patterns directly obtained from urines and in those obtained after rechromatography of the bands isolated by preparative TLC (see Methods). TLC patterns obtained after rechromatography of isolated bands showed clear ninhydrin positivity for most of the corresponding bands, including the origin. For bands 4 and 5, although positive with ninhydrin staining, the intensity was weak, as it was also for the orcinol staining. Although the rechromatographed isolated bands gave single bands after orcinol staining they do not necessarily correspond to one oligosaccharide, as only a one-step purification procedure was followed. Urinary TLC patterns from the various patients were comparable, but there was a variation in intensity of the total pattern between patients. The eight orcinol-positive bands were also visible in the pattern obtained from the urine of an infantile neuronopathic patient.

Carbohydrate composition of TLC bands: For this purpose the oligosaccharides corresponding to the eight bands were isolated from urine samples from 26 adult type 1 Gaucher patients by preparative TLC as described under Methods. The carbohydrate composition of each band revealed a high mannose, $N$-acetylglucosamine and galactose content, while $N$-acetylgalactosamine was almost absent (Table 1). Carbohydrates in these bands therefore apparently derive mainly from $N$-linked oligosaccharides.

Oligosaccharide excretion and severity of disease: Mannose content (measured by gas chromatography and expressed per mmol creatinine) was considered to be a quantitative measure for the amount of excreted oligosaccharides obtained after gel filtration. Zimran and colleagues (1992) devised a severity score index (SSI) for the objective determination of the phenotypic expression of the disease. The criteria for 
(a)



(b)

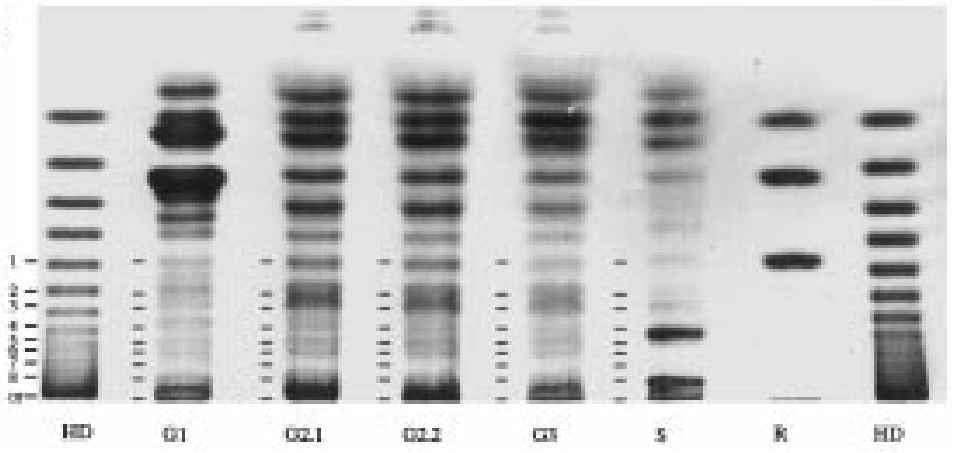

Figure 1 Thin-layer chromatography of urinary oligosaccharides. (a) Urines were desalted with Duolite mixed bed resin ion exchanger (de Jong et al 1994). HD, partially hydrolysed dextran, containing glucose monomer, dimer, trimer, etc., used as reference compound. $\mathrm{G}$, urines from adult Gaucher patients. C, urines from two different non-Gaucher individuals. (b) Oligosaccharides were partially purified by gel filtration on a Bio-Gel P2 column and chromatographed as described under Methods. HD, partially hydrolysed dextran, containing glucose monomer, dimer, trimer, etc., used as reference compound. G1, infantile neuronopathic Gaucher patient. G2.1 and G2.2, adult Gaucher patient, before (G2.1) and during (G2.2) enzyme replacement therapy. G3, adult Gaucher patient. S, urine from a sialidosis patient. R, reference compounds: glucose, lactose and sialyllactose (from top to bottom)

scoring are primarily based on the extent of organ involvement and degree of cytopenia. A positive statistically significant correlation was found between oligosaccharide-bound mannose (expressed as $\ln [$ mannose]) and the severity score index (Figure 2, Table 2). We also calculated the correlation with the independent parameters on which the SSI is based and found a positive statistically significant correlation with increase in spleen volume and a statistically significant negative correlation with haemoglobin content (Table 2). 
Table 1 Carbohydrate composition of oligosaccharide bands

\begin{tabular}{|c|c|c|c|c|c|c|c|c|c|}
\hline No. & Orcinol $^{\mathrm{a}}$ & Resorcinol $^{\mathrm{a}}$ & Ninhydrin ${ }^{\mathrm{a}}$ & Mannose $^{\mathrm{b}}$ & $G l c N A c^{\mathrm{b}, \mathrm{c}}$ & Galactose $^{\mathrm{b}}$ & $\begin{array}{l}\text { Neuraminic } \\
\text { acid }\end{array}$ & Fucose $e^{\mathrm{b}}$ & GalN $A c^{\mathrm{b}, \mathrm{c}}$ \\
\hline 1 & + & - & + & 56 & 26 & 12 & - & 1 & 4 \\
\hline 2 & $2+$ & - & $2+$ & 50 & 21 & 22 & 2 & 3 & 3 \\
\hline 3 & $2+$ & + & $2+$ & 33 & 21 & 32 & 6 & 5 & 3 \\
\hline 4 & + & - & \pm & 46 & 35 & 10 & 2.5 & 3 & 3 \\
\hline 5 & + & \pm & + & 46 & 32 & 13 & 6 & 3 & - \\
\hline 6 & $2+$ & + & \pm & 44 & 34 & 14 & 6.5 & 2 & - \\
\hline 7 & \pm & $?$ & + & 46 & 31 & 14 & 5 & 4 & - \\
\hline 8 & + & + & + & 38 & 30 & 18 & 10 & 4 & - \\
\hline Origin & $2+$ & + & $2+$ & 18 & 33 & 23 & 15 & 6 & 5 \\
\hline
\end{tabular}

${ }^{a} 2+$, clearly positive; + , positive; \pm , weakly positive; ?, dubiously positive Percentage (mol) of total per band

${ }^{\circ}$ GlucNAc, $N$-acetylglucosamine; GalNAc, $N$-acetylgalactosamine

The number of individual bands (no.) corresponds to the numbers in Figure 1 




Figure 2 Correlation between severity score index and excretion of oligosaccharide-bound mannose. Lines represent the $95 \%$ interval of confidence for individual observations

Influence of enzyme therapy on oligosaccharide excretion: Table 3 gives the relative reduction in oligosaccharide excretion before and after 6-7 and 12-14 months of enzyme replacement therapy for 3 adult type 1 non-splenectomized Gaucher patients together with the reduction in their spleen and liver volumes. As a second biochemical parameter, the relative reduction in chitotriosidase activity is given.

Table 2 Oligosaccharide excretion and severity of the disease

\begin{tabular}{lcrl}
\hline & \multicolumn{3}{c}{$\ln [$ mannose $]$} \\
\cline { 2 - 4 } & $n$ & $r$ & $p$ \\
\hline SSI & 26 & 0.61 & 0.001 \\
Increase in spleen volume & 11 & 0.79 & 0.004 \\
Increase in liver volume & 23 & 0.27 & 0.20 \\
Haemoglobin & 19 & -0.47 & 0.04 \\
Thrombocytes & 19 & -0.20 & 0.4 \\
\hline
\end{tabular}

$\ln [$ mannose $]=$ logarithm of mannose excretion

(oligosaccharide-bound)

$n=$ number of patients; $r=$ Pearson coefficient of correlation;

$p=p$-value, significance level 
Table 3 Changes in mannose excretion, spleen volume, liver volume and chitotriosidase activity during alglucerase therapy

\begin{tabular}{cccccc}
\hline Patient & $\begin{array}{c}\text { Period of } \\
\text { treatment } \\
(\text { months) }\end{array}$ & $\begin{array}{c}\text { Mannose } \\
\text { excretion } \\
\mathbf{( \% )}\end{array}$ & $\begin{array}{c}\text { Spleen } \\
\text { volume } \\
(\%)\end{array}$ & $\begin{array}{c}\text { Liver } \\
\text { volume } \\
(\%)\end{array}$ & $\begin{array}{c}\text { Chitotriosidase } \\
\text { activity } \\
(\%)\end{array}$ \\
\hline 1 & $0-7-12$ & $100-100-83$ & $100-75-66$ & $100-89-76$ & $100-91-83$ \\
2 & $0-6-12$ & $100-67-67$ & $100-65-66$ & $100-97-100$ & $100-58-51$ \\
3 & $0-6-14$ & $100-56-65$ & $100-72-59$ & $100-92-82$ & $100-85-44$ \\
\hline
\end{tabular}

\section{DISCUSSION}

The main storage product in Gaucher disease is glucosylceramide, due directly to the glucocerebrosidase deficiency. This glycolipid is mainly derived from membranes of phagocytosed leukocytes and senescent erythrocytes (DeGasperi et al 1990). Glycoprotein storage in spleen and liver from type 1 and type 3 Gaucher patients has been described by DeGasperi and colleagues (1990). Urinary excretion of sialyloligosaccharides and high-mannose-type glycoasparagines in two infantile Gaucher patients has been described by Michalski and colleagues (1983). In this investigation we detected urinary excretion of oligosaccharides and glycopeptides in adult nonneuronopathic (type 1) patients. Thin-layer chromatography patterns from the various patients were comparable with each other but showed some variation between patients in intensity of the orcinol-stained bands. Mannose and $\mathrm{N}$ acetylglucosamine were the main sugar residues. Most of the orcinol-positive bands were also ninhydrin-positive and some of them resorcinol-positive. They therefore apparently correspond to $\mathrm{N}$-glycosidic type glycopeptides and oligosaccharides derived from $\mathrm{N}$-glycosidically linked oligosaccharides. Michalski and colleagues (1983) purified and analysed sialyloligosaccharides and several glycopeptides from infantile Gaucher urine. The oligosaccharides appeared to be comparable with the $\mathrm{N}$-acetyllactosamine (complex) type oligosaccharides seen in sialidosis urines. The glycopeptides in their study contained only mannose and $\mathrm{N}$-acetylglucosamine sugar residues and were identified as glycoasparagines related to high-mannose-type carbohydrate chains of glycoproteins. Ninhydrin-positive bands analysed in our study, however, also contained galactose and some also neuraminic acid (bands 3, 5, 6 and 8). Bands isolated from the thin-layer chromatogram in our investigation probably do not correspond to homogeneous oligosaccharide or glycopeptide preparations. The galactose and neuraminic acid in ninhydrin-positive bands may be due to a combination of the glycopeptides and sialyloligosaccharides described by Michalski and colleagues (1983). There were no similarities seen between the patterns obtained from Gaucher urines and that from a sialidosis urine (Figure 1b).

Storage of glycoprotein material in spleen and liver (DeGasperi et al 1990) is apparently a secondary phenomenon due to obstruction of lysosomal enzymes, caused by the massive storage of glucosylceramide (Michalski et al 1983). DeGasperi and colleagues (1990) detected intense staining of Gaucher cells in liver and spleen with lectins that react with $N$-linked mannose- and fucose-containing oligosac- 
charide, with $\mathrm{N}$-acetyllactosamine sequences therein and with terminal galactose and neuraminic acid residues. This kind of partially degraded material might reach the circulation and will then be secreted in the form of glycopeptides.

The clinical presentation for type 1 Gaucher disease is variable and no relation can be made with biochemical parameters such as enzyme activity (van Weely et al 1991). A correlation has been demonstrated between different genotypes and the expression in Gaucher phenotypes 1 to 3 (Theophilus et al 1989; Zimran et al 1989; Beutler 1991; Sibille et al 1993). With respect to type 1 disease, symptomatic patients with genotypes other than N370S homozygosity generally have more severe disease than do N370S homozygotes (Sibille et al 1993; NIH Technology Assessment Panel on Gaucher Disease 1996). On the other hand, variation in clinical expression is seen within the same mutation group (Sidransky et al 1992; Zimran et al 1992; NIH Technology Assessment Panel on Gaucher Disease 1996), which may be due to secondary factors such as variations in activator activities, the inflow of glycolipid into the lysosomal system, or viral infection (Zimran et al 1992). There is a need for more biochemical parameters in Gaucher disease to allow characterization and prediction of progression of disease or the effect of therapy (Aerts et al 1993). We found a significantly positive correlation between oligosaccharide excretion and clinical severity of the disease expressed as severity score index (Table 2). Excretion of oligosaccharides was also significantly positively correlated with spleen size. If the excretion is caused by a secondary accumulation of glycoproteins and glycopeptides due to obstruction of the lysosomal system by an excessive amount of glycolipids, there may be a relation between oligosaccharide excretion and the amount of glycolipid stored in the body. Storage in Gaucher disease is most prominent in cells of the mononuclear phagocytic system like the spleen. Another source for the urinary glycopeptides could be the kidney. If oligosaccharide excretion indeed is a measure for the extent of storage, it would be an interesting parameter for long-term follow-up of therapy.

Table 3 shows the influence of enzyme therapy on reduction of oligosaccharide excretion, reduction of spleen and liver volume and reduction in plasma chitotriosidase activity after 6-7 and 12-14 months of therapy. Mannose excretion, spleen volume and chitotriosidase activity decreased to a similar extent. The effect of therapy on these biochemical parameters, and correlation with clinical improvement and other parameters such as regression of spleen and liver volume, haemoglobin content and platelet count will be the subjects of further investigation.

\section{ACKNOWLEDGEMENTS}

The authors thank Mrs J. van Loon and C. Wilbers, who performed the gas chromatographic analysis of oligosaccharides.

\section{REFERENCES}

Aerts JMFG, van Weely S, Boot R, Hollak CEM, Tager JM (1993) The pathogenesis of lysosomal storage disorders as illustrated by Gaucher disease. J Inher Metab Dis 16: 288291. 
Barton NW, Brady RO, Dambrosia JM et al (1991) Replacement therapy for inherited enzyme deficiency. Macrophage-targeted glucocerebrosidase for Gaucher's disease. $N$ Engl J Med 324: 1464-1470.

Beutler E (1991) Gaucher's disease. N Engl J Med 325: 1354-1360.

Beutler E, Grabowski GA (1995) Gaucher disease. In Scriver CR, Beaudet AL, Sly WS, Valle $\mathrm{D}$, eds. The Metabolic and Molecular Bases of Inherited Disease, 7th edn. New York: McGraw-Hill, 2641-2670.

Beutler E, Kay A, Saven A, et al (1991) Enzyme replacement therapy for Gaucher disease. Blood 78: 1183-1189.

Blom W, Luteijn JC, Kelholte-Dijkman H, Huijmans JG, Loonen MC (1983) Thin-layer chromatography of oligosaccharides in urine as a rapid indication for the diagnosis of lysosomal acid maltase deficiency. Clin Chim Acta 134: 221-227.

Chaplin MF (1986) Monosaccharides. In Chaplin MF, Kennedy JF, eds. Carbohydrate Analysis. A Practical Approach. Oxford: IRL Press, 23-32.

DeGasperi R, Alroy J, Richard R (1990) Glycoprotein storage in Gaucher disease: lectin histochemistry and biochemical studies. Lab Invest 63: 385-393.

de Jong J, van den Berg C, Wijburg $\mathrm{H}$ et al (1994) $\alpha$ - $N$-Acetylgalactosaminidase deficiency with mild clinical manifestations and difficult biochemical diagnosis. $J$ Pediatr 125: 385391.

Fallet S, Grace ME, Sibille A et al (1992) Enzyme augmentation in moderate to lifethreatening Gaucher disease. Pediatr Res 31: 496-502.

Gravel RA, Clarke JTR, Kaback MM (1995) The GM2 gangliosidoses. In Scriver CR, Beaudet AL, Sly WS, Valle D, eds. The Metabolic and Molecular Bases of Inherited Disease, 7th edn. New York: McGraw-Hill, 2839-2879.

Hollack CEM, van Weely S, van Oers MHJ et al (1994) Marked elevation of plasma chitotriosidase activity. A novel hallmark of Gaucher disease. J Clin Invest 93: 1288-1292.

Hollack CEM, Aerts JMFG, Goudsmit R et al (1995) Individualised low dose alglucerase therapy for type 1 Gaucher's disease. Lancet 345: 1474-1478.

Keene ON (1995) The log transformation is special. Stat Med 14: 811-819.

Michalski JC, Montreuil J, Strecker G (1983) Primary structure of three mannosylglycoasparagines and nine sialyl-oligosaccharides isolated from the urine of two patients with Gaucher's disease (infantile form). Eur J Biochem 132: 375-381.

NIH Technology Assessment Panel on Gaucher Disease (1996) Gaucher disease. Current issues in diagnosis and treatment. $J$ Am Med Assoc 275: 548-553.

Pastores GM, Sibille AR, Grabowski GA (1993) Enzyme therapy in Gaucher disease type 1: dosage efficacy and adverse effects in 33 patients treated for 6 to 24 months. Blood 82 : 408-416.

Schindler D, Kanzaki T, Desnick RJ (1990) A method for the rapid detection of urinary glycopeptides in $\alpha-N$-acetylgalactosaminidase deficiency and other lysosomal storage diseases. Clin Chim Acta 190: 81-92.

Sibille A, Eng CM, Kim SJ, Pastores G, Grabowski GA (1993) Phenotype/genotype correlations in Gaucher disease type I: clinical and therapeutic implications. Am J Hum Genet 52: 1094-1101.

Sidransky E, Tsuji S, Martin BM, Stubblefield B, Ginns EI (1992) DNA mutation analysis of Gaucher patients. Am J Med Genet 42: 331-336.

Suzuki Y, Sakuraba H, Oshima A (1995) $\beta$-Galactosidase deficiency ( $\beta$-galactosidosis): GM1 gangliosidosis and Morquio B disease. In Scriver CR, Beaudet AL, Sly WS, Valle D, eds. The Metabolic and Molecular Bases of Inherited Disease, 7th edn. New York: McGrawHill, 2785-2823.

Theophilus B, Latham T, Grabowski GA (1989) Gaucher disease: molecular heterogeneity and phenotype-genotype correlations. Am J Hum Genet 45: 212-225.

Thomas GH, Beaudet AL (1995) Disorders of glycoprotein degradation and structure: $\alpha$ mannosidosis, $\beta$-mannosidosis, fucosidosis, sialidosis, aspartylglucosaminuria and carbohydrate deficient glycoprotein syndrome. In Scriver CR, Beaudet AL, Sly WS, Valle D, eds. 
The Metabolic and Molecular Bases of Inherited Disease, 7th edn. New York: McGrawHill, 2529-2561.

van Weely S, van Leeuwen MB, Jansen IDC et al (1991) Clinical phenotype of Gaucher disease in relation to properties of mutant glucocerebrosidase in cultured fibroblasts. Biochim Biophys Acta 1096: 301-311.

Zimran A, Sorge J, Grosse E (1989) Prediction of severity of Gaucher's disease by identification of mutations at DNA level. Lancet 2: 349-352.

Zimran A, Kay A, Gelbart T (1992) Gaucher disease. Clinical, laboratory, radiologic, and genetic features of 53 patients. Medicine 71: 337-353. 